\title{
Strongyloides stercoralis como causa infrecuente de infección de líquido ascítico en cirrosis
}

\author{
Strongyloides stercoralis as uncommon cause of ascitic fluid infection in cirrhosis
}

\author{
Amaya Ortiz y María Moreno ${ }^{1}$
}

${ }^{1}$ Hospital de Clínicas, San Lorenzo, Paraguay.

Los autores declaramos no tener conflicto de interés alguno.

No hubo financiación externa para este trabajo.

Recibido: 10 de junio de 2019 / Aceptado: 7 de octubre de 2019

\section{Resumen}

Se comunica el caso clínico de un varón, con antecedentes de una cirrosis hepática alcohólica y gota, usuario crónico de antiinflamatorios, incluyendo corticoesteroides. Consultó por una melena secundaria a una úlcera bulbar. Durante su internación presentó fiebre, tratándose con ceftriaxona por un probable foco urinario. Por persistir febril, se realizó una paracentesis diagnóstica. En la muestra de líquido ascítico se observaron larvas de Strongyloides stercoralis. Recibió tratamiento antiparasitario con ivermectina, con buena respuesta clínica. Aunque la infección por S. stercoralis es relativamente frecuente en pacientes con cirrosis hepática alcohólica, la ascitis infectada por Strongyloides corresponde a una forma de presentación infrecuente. Este caso muestra la importancia de la paracentesis diagnóstica en todo paciente con ascitis secundaria a una cirrosis. Es importante considerar la presentación atípica de la infestación por Strongyloides en el contexto del paciente inmunocomprometido, ya que sin tratamiento puede tener una alta mortalidad.

Palabras clave: Strongyloides stercoralis; ascitis.

\section{Introducción}

$S$ trongyloides stercoralis es un parásito nematodo endémico en algunas zonas de Latinoamérica, Asia y África, que provoca infecciones de manifestación clínica muy variada: desde eosinofilia asintomática, cuadros gastrointestinales o respiratorios poco específicos, hasta cuadros graves como la infección diseminada y el shock séptico. En la presentación de esta patología, influye el estado

\begin{abstract}
Male patient, with a history of alcoholic cirrhosis frequent user of anti-inflammatory drugs including corticosteroids. He consulted for digestive bleeding secondary to a bulbar ulcer. During the admission, he had fever and antibiotic treatment with ceftriaxone is started, for a urinary infection. Fever persisted for 48 hours, so a diagnostic paracentesis was made: Strongyloides stercoralis larvae were seen in the direct microscopic exam. The patient started antiparasitic treatment with ivermectin. He was discharged and did not returned for follow up. Although infection with $S$. stercoralis is relatively common in patients with alcoholic liver cirrhosis, ascites infected with Strongyloides corresponds to an infrequent form of presentation. This case shows the importance of diagnostic paracentesis in every cirrhotic patient. It is important to consider atypical presentation of Strongyloides infection in the immunocompromised host, considering it could be fatal without treatment.
\end{abstract}

Keywords: Strongyloides stercoralis; ascitic fluid.

inmunitario del paciente, y para el diagnóstico se debe tener una alta sospecha clínica. Las manifestaciones clínicas tan variadas, sobre todo en el contexto del paciente inmunocomprometido, frecuentemente llevan a un retraso en el diagnóstico y tratamiento de la infección por este parásito. Comunicamos el caso clínico de un paciente con cirrosis hepática alcohólica, consumidor crónico de corticoesteroides, con una localización inusual del parásito: infección del líquido ascítico por S. stercoralis. 


\section{Caso clínico}

Varón de 55 años, procedente de Paraguay. Con antecedentes de ser consumidor excesivo de alcohol y tabaco, de más de $120 \mathrm{~g}$ a la semana de etanol, portador de artritis gotosa, consumidor crónico de antiinflamatorios no esteroidales y corticoesteroides. Consultó por melena de varias semanas de evolución, sin inestabilidad hemodinámica. Ingresa al Servicio de Urgencia lúcido, afebril, hemodinámicamente estable. Al examen físico, destacaba una palidez intensa; además, se constataron arañas vasculares, circulación colateral, eritema palmar y ascitis moderada-abundante, como signos de hepatopatía crónica.

Dentro de los exámenes de laboratorio del ingreso destacaba una hemoglobina de $3,5 \mathrm{~g} / \mathrm{dl}$, hematocrito $10 \%$, leucocitos 16.900 céls $/ \mathrm{mm}^{3}$, neutrófilos $85 \%$, linfocitos $11 \%$, monocitos $3 \%$ y eosinófilos $1 \%$. El perfil hepático era normal. Las serologías virales (IgM para hepatitis A, hepatitis C, VIH), y el HbsAg fueron negativos.

Fue sometido a una endoscopía digestiva alta, constatándose una pangastritis eritematosa y una-úlcera bulbar Forrest III. Se extrajeron muestras de biopsias para estudio de Helicobacter pylori. Recibió tratamiento con un inhibidor de la bomba de protones; además de transfusión de hemoderivados. Posterior al cese de la melena y a las transfusiones, la hemoglobina se mantuvo estable, en alrededor de $8 \mathrm{~g} / \mathrm{dl}$.

Se realizó una ecografía doppler abdominal que confirmó la hepatopatía crónica, así como hipertensión portal y ascitis importante.

Durante su internación en sala, presentó un episodio febril de $38,2^{\circ} \mathrm{C}$, con sedimento de orina alterado, aunque sin síntomas urinarios típicos. Se inició terapia antibacteriana con ceftriaxona $2 \mathrm{~g}$ al día. Al tercer día de tratamiento, presentó nuevamente fiebre. No había cambios al examen físico, ni síntomas respiratorios o urinarios. Se realizó una paracentesis diagnóstica; el análisis del líquido ascítico, ya con más de 48 h de tratamiento antibacteriano, era ligeramente turbio; proteínas $1,1 \mathrm{mg} / \mathrm{dl}$, gradiente albúmina suero-líquido ascítico de 1,$4 ; 104$ leucocitos $/ \mathrm{mm}^{3}, 60 \%$ polimorfonucleares y $40 \%$ mononucleares. En el examen directo del extendido, se observaron larvas de $S$. stercoralis (Figuras 1, 2 y 3). El cultivo corriente para bacterias resultó negativo. El estudio copro-parasitológico seriado de heces, también fue negativo.

Se decidió continuar la terapia antibacteriana, agregándose tratamiento antiparasitario con ivermectina a $0,2 \mathrm{mg} / \mathrm{kg} /$ día por 5 días, con resolución de la fiebre. El paciente fue dado de alta y no retornó para seguimiento.

\section{Discusión}

La infección por S. stercoralis es relativamente frecuente en pacientes cirróticos. Casi un tercio de ellos tienen copro-parasitológico positivo. Inclusive su prevalencia es mayor si está asociado a etilismo $(44 \%)^{1,2}$.

Las manifestaciones clínicas de esta parasitosis son muy variadas: desde eosinofilia asintomática hasta la enfermedad diseminada y shock séptico; particularmente la ascitis infectada por Strongyloides corresponde a una forma de presentación infrecuente, descrita en la literatura médica en casos esporádicos ${ }^{3-6}$.

En este caso, se sospechó una infección por S. stercoralis por la persistencia de fiebre en un paciente con tratamiento antibacteriano por una dudosa infección
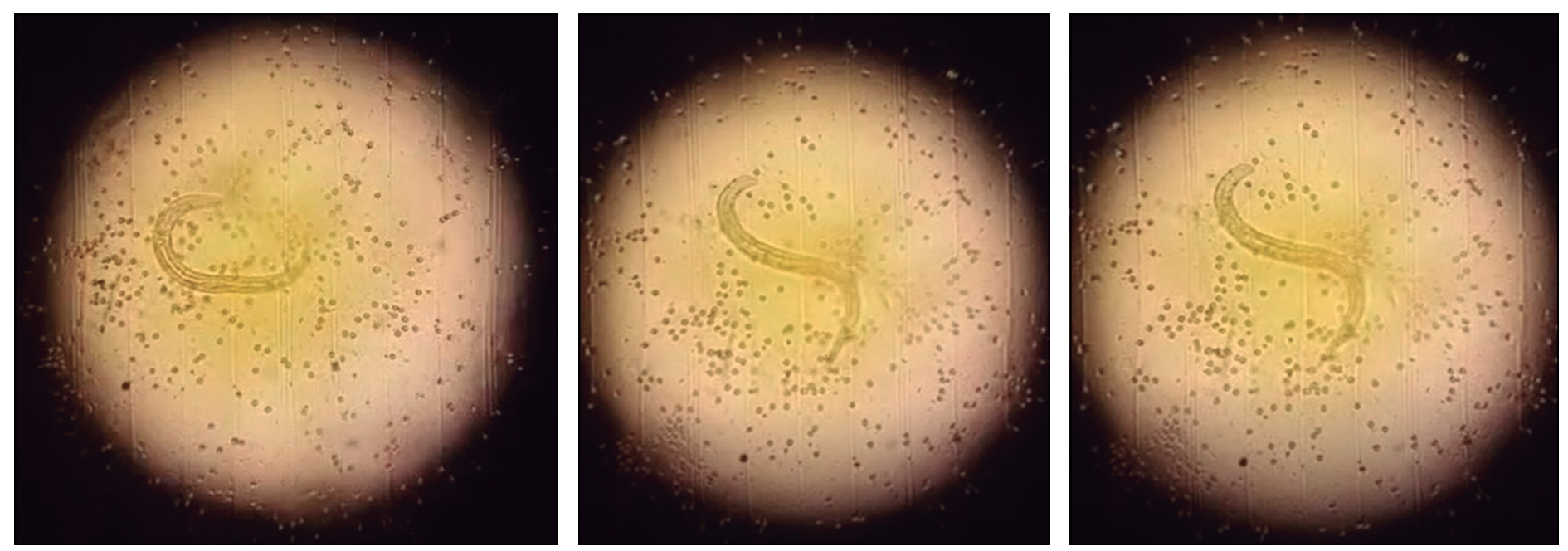

Figuras 1, 2 y 3. Visión microscópica de larvas de Strongiloydes stercoralis en el examen directo del líquido ascítico. 
urinaria. El líquido ascítico no resultó compatible con una peritonitis bacteriana espontánea. Sin embargo, se debe considerar que éste fue estudiado al tercer día de terapia antibacteriana, por lo que existe la posibilidad de que inicialmente haya habido una peritonitis bacteriana que respondió al tratamiento con cefalosporinas, descendiendo el conteo de neutrófilos del líquido ascítico a menos de 250 por campo. En este caso, Strongyloides pudo haber actuado como vector, lo que ha sido descrito en la literatura?

El paciente, consumidor excesivo de alcohol, con antecedentes de cirrosis hepática y uso crónico de corticoesteroides, presenta varios factores predisponentes para la infección diseminada por S. stercoralis. Llama la atención la ausencia de eosinofilia periférica, que podría estar relacionada con el grado de inmunocompromiso y consecuente supresión de eosinófilos, por el uso crónico de corticoesteroides? ${ }^{7}$. El resultado del copro-parasitológico seriado fue negativo para Strongyloides, aunque debe mencionarse que la técnica usada fue el examen directo: técnica sencilla y de uso muy frecuente, pero de bajo rendimiento al compararse con otras, como la placa de ágar o la técnica de Baermann ${ }^{8}$. De todos modos, el examen directo del líquido ascítico fue suficiente.

Este caso muestra la importancia de la paracentesis diagnóstica en todo paciente cirrótico al momento de buscar un foco infeccioso, a pesar de la ausencia de otros signos clínicos como el dolor abdominal. El tratamiento iniciado por la sospecha de un foco urinario, tenía una buena cobertura también para una probable peritonitis bacteriana espontánea.

Es importante considerar la presentación atípica de la infestación por Strongyloides en el contexto de un paciente inmunocomprometido, en este caso secundario a una cirrosis hepática y al uso de corticooesteroides, ya que sin tratamiento puede llegar tener una alta mortalidad. Lamentablemente, en este caso si bien se constató una mejoría clínica con el tratamiento instaurado, debido al abandono del control, no se pudo comprobar la eliminación del parásito.

\section{Referencias bibliográficas}

1.- Teixeira M, Pacheco F, De Souza J, Silva M, Ines E, Soares N. Strongyloides stercoralis infection in alcoholic patients. Biomed Res Int 2016; 1: 1-11. doi: 10.1155/2016/4872473.

2.- de Oliveira L C, Ribeiro CT, Mendes D de M, Oliveira T C, Costa-Cruz J M. Frequency of Strongyloides stercoralis infection in alcoholics. Mem Inst Oswaldo Cruz 2002; 97 : 119-21.

3.- André M H, Oshiro Bansho E T, Tonon D, Vieira E, Vieira ML, Dantas-Correa E B, et al. An unusual cause of ascites: Strongyloides stercoralis. J Clin Gastroenterol 2014; 48: 3012. doi: 10.1097/MCG.0b013e3182aa1184.

4.- Shukla S, Chauhan R, Wadhwa S, Sehgal S, Singh S. Strongyloides stercoralis hyperinfection causing eosinophilic ascites. Diagn Cytopathol 2015; 43:731-3. doi: $10.1002 / \mathrm{dc} .23281$.

5.- Jariwala S, Langman Y, Benson A, Wolf E, Moss J, Zhu C C, et al. Strongyloidiasis presenting as eosinophilic ascites. Ann Trop Med Parasitol 2011; 105:335-8. doi: 10.1179/13 6485911X12987676649863.
6.- Hong I S, Zaidi S Y, McEvoy P, Neafie R C. Diagnosis of Strongyloides stercoralis in a peritoneal effusion from an HIV seropositive man. A case report. Acta Cytol 2004; 48: 211-4.

7.- Vadlamudi R S, Chi D S, Krishnaswamy G. Intestinal strongyloidiasis and hyperinfection syndrome. Clin Mol Allergy 2006; 4: 8.

8.- Campo Polanco L, Gutiérrez L A, Cardona Arias J. Infección por Strongyloides stercoralis: metanálisis sobre evaluación de métodos diagnósticos convencionales (1980-2013). Rev Esp Salud Publica 2014; 88: 581-600. doi: 10.4321/S1135-57272014000500004. 